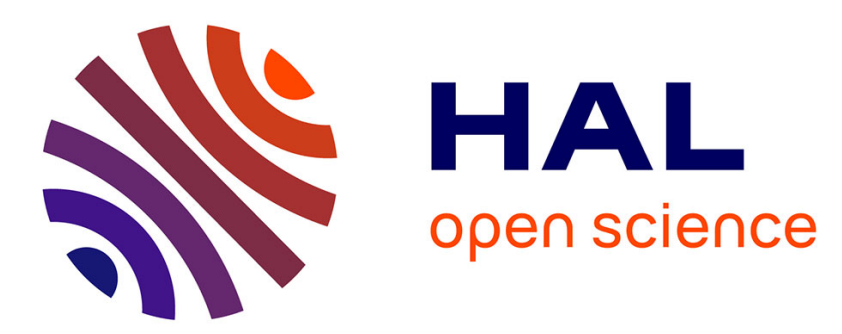

\title{
Casque antibruit actif basses-fréquences et audition dans le bruit
}

\author{
J.-C. Béra, C. Micheyl, L. Collet
}

\section{To cite this version:}

J.-C. Béra, C. Micheyl, L. Collet. Casque antibruit actif basses-fréquences et audition dans le bruit. Journal de Physique IV Proceedings, 1994, 04 (C5), pp.C5-391-C5-394. 10.1051/jp4:1994581 . jpa00253076

\section{HAL Id: jpa-00253076 https://hal.science/jpa-00253076}

Submitted on 1 Jan 1994

HAL is a multi-disciplinary open access archive for the deposit and dissemination of scientific research documents, whether they are published or not. The documents may come from teaching and research institutions in France or abroad, or from public or private research centers.
L'archive ouverte pluridisciplinaire HAL, est destinée au dépôt et à la diffusion de documents scientifiques de niveau recherche, publiés ou non, émanant des établissements d'enseignement et de recherche français ou étrangers, des laboratoires publics ou privés. 


\title{
Casque antibruit actif basses-fréquences et audition dans le bruit
}

\author{
J.-C. BÉRA, C. MICHEYL* et L. COLLET*
}

URA 263 du CNRS, Centre Acoustique de l'E.C.L., 36 avenue de Collongue, BP. 163, 69131 Ecully cedex, France

"URA 1447 du CNRS, Laboratoire de Physiologie Sensorielle "Audition et Voix", HEH Pavillon U, 3 place d'Arsonval, 69437 Lyon cedex 03, France

\begin{abstract}
A low-frequency active anti-noise headset has been made, and tested on human ears with regard to hearing in noise. Psychoacoustic methods are used in order to quantify the improvement in audibility and intelligibility. Under low-frequency noise, a spectacular effect of anti-noise is observed, while headset wearing brings just a very little global improvement because of negative passive effect. Besides optimization of passive acoustic attenuation, evaluation of such an apparatus is faced with interindividual hearing in noise variability.
\end{abstract}

\section{INTRODUCTION}

L'audibilité en milieu bruyant (usine, voiture, train, avion, salles communes) constitue à l'heure actuelle un probleme de communication essentiel, qui concerne autant le secteur industriel que le confort de la vie quotidienne. Outre la lutte contre les sources de bruit elles-mêmes, des méthodes locales peuvent être proposées : isolation acoustique, optimisation de la réflexion et de l'absorption par les parois, antibruit référencé à la rotation des moteurs, systèmes de protection individuel.

Sur ce dernier point, plusieurs études ultérieures ont testé des casques antibruits passifs vis-à-vis de l'amélioration de l'intelligibilité : des résultats positifs sont observés pour certains types de bruits de fond de forte intensité. Certains casques se basent sur un fonctionnement non-linéaire ${ }^{1}$ ), en présentant une atténuation qui augmente avec le niveau sonore de stimulation : ce qui permet de compresser un peu la gamme d'intensités vers les intensités pour lesquelles l'oreille est la plus sélective. Par ailleurs, l'atténuation peut être faible sur les principales composantes de la parole.

Cependant, dans la mesure où tous ces casques passifs ne présentent pas ou peu d'atténuation visà-vis des sons graves, le masquage de la parole par des bruits basses-fréquences ne peut être évité. Au contraire, les systèmes d'antibruit actif se trouvent particulièrement adaptés au traitement des basses fréquences. A noter qu'il existe des casques électroniques (couplés à une atténuation passive) qui amplifient les fréquences moyennes $(500$ à $2500 \mathrm{~Hz})(2)$, afin de favoriser les signaux de parole : efficaces pour une gamme d'intensités de bruits très restreinte, ces systèmes actifs présentent en outre le désavantage d'augmenter le niveau sonore global.

En tout état de cause, une appréciation subjective de l'effet de l'antibruit actif dans un environnement bruyant basses-fréquences fait conclure à une possibilité d'action positive vis-à-vis de l'audibilité de la parole, amélioration qu'il conviendrait cependant de quantifier. Le but de cette étude est précisément d'effectuer des mesures de l'amélioration de l'audibilité apportée par un casque antibruit actif, en cherchant à mettre en évidence les paramètres essentiels susceptibles d'améliorer le procédé vis-à-vis de l'intelligibilité dans le bruit, ainsi que les problèmes de test de cette efficacité.

(1) ex : casque "ULTRA 9000" de EAR, avec son dispositif à valve.

(2) ex : casque électronique "BILSOM COM IMPACT" de BILSOM. 


\section{CASOUE ANTIBRUTT ACTIF BASSES-FRÉQUENCES}

\section{Problématique :}

L'idée générale consiste à diminuer le rapport signal utile sur bruit, non pas en amplifiant le signal utile, mais en diminuant le niveau du bruit masquant. On rappelle deux avantages à diminuer le niveau acoustique global d'un bruit de forte intensité : minimiser la stimulation acoustique de l'oreille (pour prévenir traumatismes sonores et fatigue), favoriser l'efficacité auditive (puisque la non-linéarité de la perception entraîne un regain de sélectivité aux niveaux moyens de stimulation).

La présente étude concerne plus précisément la discrimination de la parole en milieu bruyant. Dans le principe, on cherche à discriminer le signal utile, du bruit, dans l'espace fréquentiel : on accepte donc de ne pas agir sur le rapport signal sur bruit dans le cas où le bruit masquant et le signal utile se manifestent aux mêmes fréquences. Pour ce faire, on suppose que le signal utile de la parole se trouve principalement concentré sur des fréquences supérieures à $400 \mathrm{~Hz}$. Au niveau du bruit de fond ambiant, on va s'intéresser plus particulièrement à un bruit basses-fréquences. De tels bruits se rencontrent en effet fréquemment en tant que sons masquant : ainsi, typiquement, le bruit de bourdonnement des moteurs à explosion, qui comporte de fortes composantes basses fréquences.

\section{Système utilisé :}

\section{Réalisation pratique :}

Le casque antibruit actif utilisé, monté sur un casque audiophonique semi-ouvert (SONY CD250), comporte deux systèmes identiques et parfaitement séparés (un par oreille). Leur fonctionnement est basé sur une minimisation locale de la pression acoustique, réalisée selon le principe de Carme [1] : pour chaque oreille, le son est mesuré à l'entrée du conduit auditif externe par un microphone, le signal est traité par un filtre analogique (à deux pôles et deux zéros), puis restitué dans l'écouteur du casque. Tout en assurant les conditions de stabilité et d'atténuation, le filtre coupe les moyennes et hautes fréquences.

\section{Performances du système actif :}

Dans ce qui suit, les résultats sont présentés pour deux gains de la boucle d'antibruit: correspondant à ce qu'on appelle le réglage $\mathrm{A}$ du casque, et le réglage $\mathrm{B}$ ( $6 \mathrm{~dB}$ de plus que $\mathrm{A})$. Nous avons donc réalisé un casque antibruit basses-fréquences : en réglage $\mathrm{B}$, l'atténuation est de l'ordre de 15 $\mathrm{dB}$ pour les fréquences inférieures à $400 \mathrm{~Hz}$, tandis que le gain acoustique du système actif est voisin de 1 au-delà de $400 \mathrm{~Hz}$ (fig.1).

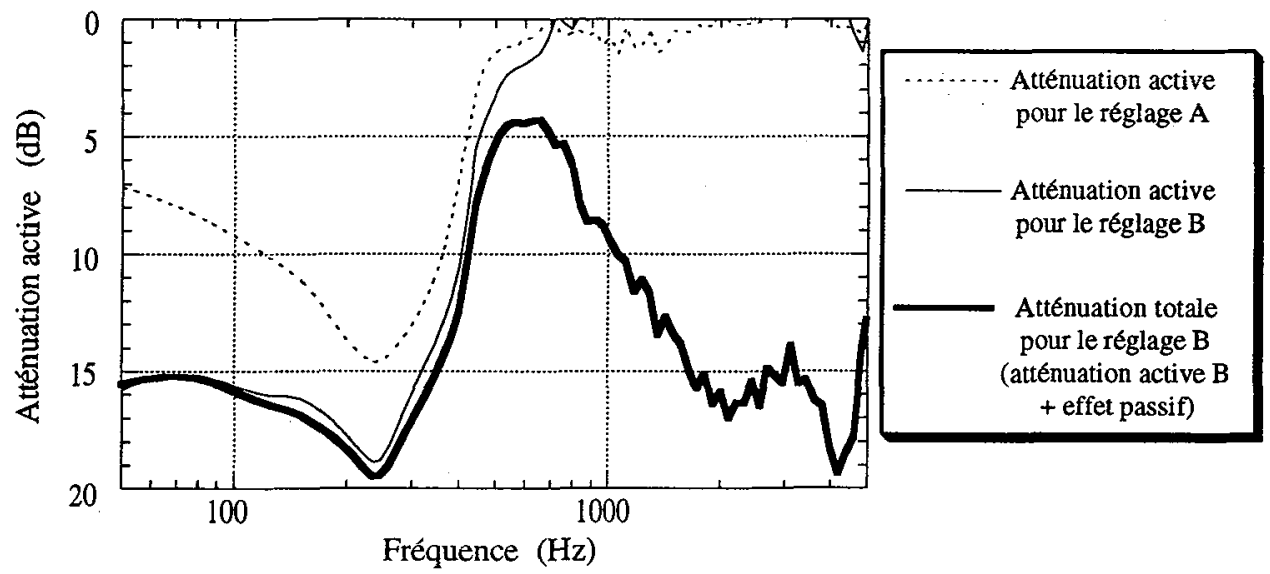

Figure 1 - Courbes fréquentielles d'atténuation du casque considéré. 


\section{TESTS PSYCHOACOUSTIQUES: mesure de l'amélioration de l'audibilité dans le bruit}

Ces tests ont été réalisés de façon extensive sur deux sujets normo-entendants, choisis pour présenter les mêmes "caractéristiques acoustiques" : même fonction de transfert électroacoustique et même atténuation mesurée par le microphone du casque (fig.1).

\section{Seuil d'audition de sons purs dans le bruit :}

Afin d'obtenir une mesure quantitative de l'effet du casque antibruit sur les performances auditives, les seuils absolus de perception de sons purs en champ libre sont successivement testés dans trois conditions $: 1^{\circ}$ ) sans casque, $2^{\circ}$ ) avec le casque éteint (i.e. casque passif uniquement), $3^{\circ}$ ) avec le casque allumé. Dans cette dernière condition, pour mettre en évidence une graduation des effets, 2 réglages du casque sont utilisés ( $\mathrm{A}$ et $\mathrm{B}$, cf. fig 1 ).

La mesure des seuils est effectuée au moyen d'une procédure psychophysique classique [2] : la méthode adaptative à 2 intervalles en choix forcé. Afin de garantir une fiabilité suffisante des mesures, a été choisie une règle d'adaptation 3 down / 1 up - consistant à incrémenter le niveau après chaque réponse fausse et à ne le décrémenter qu'après 3 réponses correctes successives. Le pas, initialement de $5 \mathrm{~dB}$, est réduit à $1 \mathrm{~dB}$ après les trois premières inversions. Au terme des 70 essais de chaque mesure, une évaluation du seuil est obtenu comme moyenne des niveaux atteints lors des dernières inversions.

Les stimuli consistent en des tone-pips de $100 \mathrm{~ms}$ intervenant $100 \mathrm{~ms}$ après le début d'un bruit de fond de $300 \mathrm{~ms}$; pour minimiser les transitoires hautes-fréquences, les signaux ont des temps de montée et descente relativement longs $(20 \mathrm{~ms})$. L'audibilité est testée aux fréquences $250,500,1000,2000$ et $4000 \mathrm{~Hz}$, tandis que le bruit masquant consiste en un son aléatoire à bande étroite basses-fréquences de 100 à $400 \mathrm{~Hz}$ et de $85 \mathrm{~dB}$ SPL. Les signaux sont générés après FFT inverse par un convertisseur D/A 16 bits, avec une fréquence d'échantillonnage de $40 \mathrm{kHz}$. L'ensemble de l'expérimentation a été mise en oeuvre sur un poste de psychoacoustique constitué par un AT286 équipé du matériel Tucker Davies Technologies (carte D/A, atténuateurs programmables) et du logiciel Xperimenter.

La méthode utilisée fournie des résultats quantitatifs très précis et parfaitement reproductibles. On constate que le casque utilisé en réglage $B$ apporte une amélioration notable de l'audibilité des sons purs : le seuil d'audibilité est abaissé de plus de $5 \mathrm{~dB}$ pour les sons de fréquences 500 et $1000 \mathrm{~Hz}$, tandis qu'il est peu modifié pour les sons de 250 et $4000 \mathrm{~Hz}$ (fig.2). En outre l'atténuation passive du casque pénalise fortement ces seuils : ainsi, le système actif à lui seul apporte une amélioration de plus de $10 \mathrm{~dB}$ à 500 et $1000 \mathrm{~Hz}$ (différence entre "casque OFF" et "réglage B").

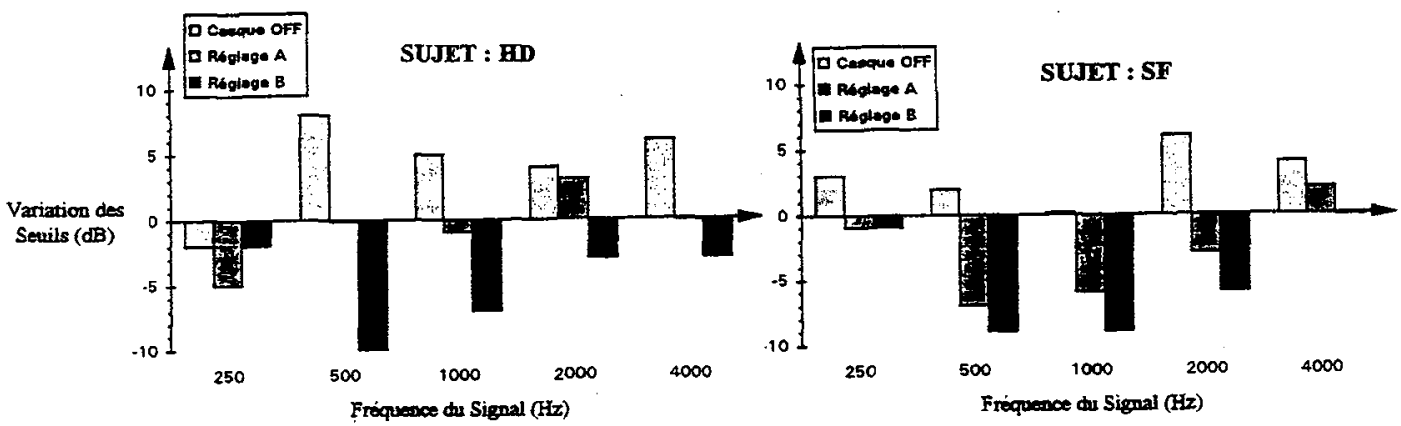

Figure 2 - Variations du seuil d'audibilité par rapport à l'audition sans casque, pour les sujets HD et SF.

\section{Intelligibilité de la parole dans le bruit :}

Afin d'obtenir des indications sur les effets introduits par le casque concernant les capacites de compréhension de la parole dans le bruit, on a utilisé une procédure inspirée des techniques d'audiométrie vocale : le décompte du pourcentage de phonèmes correctement identifiées au sein de mots de 3 phonèmes (listes de Laffont). Selon la procédure clinique conventionnelle en audiométrie vocale, les mots sont prononcés au micro par un locuteur contrôlant le niveau moyen de sa voix au moyen d'un VU-mètre. Au terme d'essais préliminaires, une intensité de $20 \mathrm{~dB}$ au-dessus du seuil individuel de perception dans le bruit est choisie, de façon à rendre difficile, mais possible, l'identification des mots. Le bruit masquant est ici un signal continu basses-fréquences (de 100 à $400 \mathrm{~Hz}$ ) et de $85 \mathrm{~dB}$ SPL. 
Dans l'expérience réalisée, le système actif ne parvient qu'à compenser la perte d'intelligibilité due à l'effet passif du casque (figure 3).

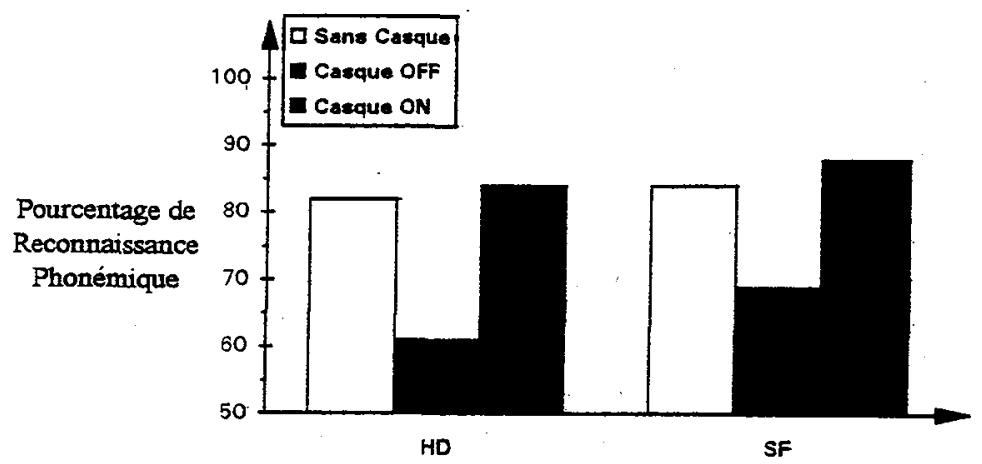

Figure 3 - Pourcentage de reconnaissance phonémique par les sujets HD et SF : sans casque, avec casque éteint (effet passif) et avec casque actif en fonctionnement

\section{DISCUSSION}

\section{Optimisation conjointe des atténuations active et passive :}

Dans les tests présentés ici, l'atténuation passive du casque a présenté globalement un effet négatif vis-à-vis de l'audibilité. Pour lever ce problème, un autre casque antibruit, présentant les mêmes performances d'atténuation active mais une très faible atténuation passive, a été réalisé à partir d'un casque audiophonique ouvert (Sennheiser HD480). Cependant, il ne faut pas perdre de vue que si le bruit masquant présente des composantes plus hautes-fréquences, l'effet passif peut être très favorable : ce qui, en l'absence d'a priori sur la nature du bruit masquant, met en évidence l'insuffisance de nos tests vis-à-vis de l'évaluation de l'efficacité d'un système donné. De sorte que la configuration du casque en vue de l'amélioration de l'intelligibilité doit résulter d'un compromis entre : une forte atténuation hautesfréquences (atténuation passive) et basses fréquences (principalement active), une faible atténuation relative des fréquences vocales, et d'une atténuation globale plus ou moins importante selon le niveau du bruit à contrer.

\section{Variabilité interindividuelle - Complexité de l'audition dans le bruit :}

Outre le problème de variabilité interindividuelle de fonctionnement électro-acoustique du casque, qui est en grande part éludé ici (puisque les 2 sujets présentés sont choisis pour l'exacte similitude de leur comportement acoustique vis-à-vis du casque), on observe des résultats très différents pour les 2 sujets. En effet les mécanismes physiologiques d'audition dans le bruit sont complexes, et pourraient mêmes différer légèrement selon les individus. En tout état de cause, la variabilité interindividuelle considérable vis-à-vis de l'interprétation d'un signal acoustique bruité constitue un obstacle majeur à l'évaluation quantitative objective de l'efficacité du procédé : en d'autre termes, la comparaison de deux casques différents ne pourra se faire que pour un individu donné ou pour un individu moyen, et pour un environnement sonore donné ou moyen.

\section{CONCLUSION}

On a mesuré de façon quantitative une amélioration importante de l'audition dans le bruit par un casque antibruit actif basses-fréquences. Cependant, la forte variabilité interindividuelle vis-à-vis de l'audition dans le bruit accrô̂t encore la difficulté à évaluer quantitativement l'efficacité d'un tel appareil.

[1] Carme C., "Absorption acoustique active dans les cavités auditives", Acustica 66 (1988), 233-246.

[2] Levitt H., "Transformed up-down methods in psychoacoustics", Journal of the Acoustical Society of America 49 (1970), 467-476. 